\title{
Problèmes des chocs de bateaux sur les ouvrages
}

\author{
Impact loads of ships on structures
}

\section{P. Dubois, P. Cornier et J. Mamet}

Compagnie Nationale du Rhône, Lyon

\section{Corbic}

Service Central des Ports Maritimes et des Voies Navigables, Compiègne

\section{I - DISPOSITIFS DE SECURITE DANS LES ECLUSES}

Rappel des différents dispositifs de sécurité installés

Pour éviter tout arrêt intempestif du trafic sur les écluses du Rhône, la Compagnie Nationale du Rhône a été amenée à protéger les équipements essentiels des ouvrages (portes et masque aval), afin d'éviter leur dégradation consécutive au choc direct des embarcations.

Ces protections comprennent :

- un dispositif de sécurité amont constitué par un organe d'arrêt (câble ou chaîne) tendu en travers de la passe, en amont de la porte.

Le dernier dispositif installé (écluse de Vaugris) est capable d'arrêter un bateau possédant une énergie cinétique de $10^{3}$ kilojoules. Les dispositif amortisseur peut fonctionner pour des bateaux heurtant le dispositif jusqu'à une vitesse de $3 \mathrm{~m} / \mathrm{s}$, la course de freinage étant limitée à $6 \mathrm{~m}$.

Les mécanismes de manœuvre du dispositif de sécurité permettent l'abaissement de l'organe d'arrêt avec ses supports lors du passage des bateaux et ensuite la remise en position normale. Ces manœuvres sont exécutées simultanément sur les deux rives de l'écluse, mais sans dispositif de synchronisation, la remise à niveau se faisant uniquement en position basse.

Le dispositif est levé ou abaissé dans un temps n'excédant pas une minute. Il suit également les variations du plan d'eau amont, afin que l'organe d'arrêt se trouve à $1,00 \mathrm{~m}$ environ au-dessus du plan d'eau.

Le dispositif comporte également un système de dévasage des rainures (deux vannes de diamètre $300 \mathrm{~mm}$ ) au moyen d'eau sous pression.
- un pare-choc aval qui protège le masque aval pour les anciennes écluses, la porte aval pour les écluses plus récentes, du choc direct des bateaux avalants entrant dans le sas.

Il convient de noter qu'en 1966, la décision a été prise en accord avec le Service de la Navigation, de ne plus installer de dispositif de sécurité aval.

Par suite des dispositions générales adoptées sur nos écluses, les bateaux montants stationnent assez loin en aval, ou restent même dans le courant du canal de fuite. Etant au surplus bien protégés du vent, les bateaux gouvernent sans difficulté.

Il faut d'ailleurs souligner que le dispositif de sécurité aval était très sensible aux dépôts de limon que l'on constate dans le bief et dans les rainures. Bien qu'il fût possible d'exécuter des chasses, il arrivait fréquemment que ce dispositif soit en dérangement, la sécurité des écluses n'en étant pas pour autant affectée.

Pour toutes ces raisons, l'installation d'un dispositif de sécurité aval n'a pas paru indispensable pour la sécurité des écluses du Rhône, et il a été déposé dans les écluses qui en étaient équipées.

On peut noter également que dans les écluses du Rhône, le risque de choc d'un bateau montant contre la porte amont n'est pas possible pour les automoteurs et convois poussés du fait de la présence du mur de chute. Par contre, pour les cargos fluviaux maritimes qui sont beaucoup plus hauts sur l'eau, et dont la proue est effilée, ce risque n'est pas totalement exclu.

Nouveaux dispositifs pare-chocs aval

\section{Evolution des gabarits sur le Rhône}

En 1942, le projet du premier aménagement sur le Bas-Rhône, en l'occurrence Donzère-Mondragon, com- 
portait une écluse unique de $195 \mathrm{~m}$ de longueur utile et $12 \mathrm{~m}$ de largeur. Ces dimensions devaient permettre l'éclusage d'un convoi constitué de 2 chalands et d'un remorqueur à hélice de 1000 ch d'une quarantaine de mètres de long.

Or, ce type de remorqueur n'existait pas sur le Rhône et seuls naviguaient des remorqueurs à roue, datant de 1913, d'une puissance de $1500 \mathrm{ch}$ et d'une largeur de $20 \mathrm{~m}$ pouvant tirer 3 chalands.

La C.N.R. a estimé qu'ils seraient prochainement remplacés par des remorqueurs à hélice d'une largeur inférieure à $12 \mathrm{~m}$, mais l'Administration n'a accepté cette largeur de $12 \mathrm{~m}$ pour le sas qu'après les essais de navigation sur le Rhône d'un remorqueur à hélice de $9 \mathrm{~m}$ de large et de $2200 \mathrm{ch}$, le "Frédéric-Mistral-RhinRhône", construit par la C.N.R.

Après la construction de l'écluse, les usagers du Rhône se sont adaptés immédiatement aux conditions nouvelles : ce fut l'arrivée effective d'un remorqueur de 1200 ch pouvant tirer 2 chalands.

Ces dimensions de $195 \times 12 \mathrm{~m}$ furent reconduites pour toutes les écluses des aménagements du Bas-Rhône, bien que le remorquage dispant pour laisser le champ libre aux automateurs à hélice de dimensions inférieures.

En 1975, la période des seuls automoteurs se termine avec l'arrivée des convois poussés. Pour ce nouveau type de bâtiments, ladaptation des usagers du Rhône, aux conditions de navigation qui leur sont offertes, s'est également manifestée puisque les dimensions des convois sont maintenant de $190 \times 11,40 \mathrm{~m}$.

L'arrivée de ces convois poussés a conduit la Compagnie à revoir complètement les dispositifs pare-chocs des écluses de Bollène et Chateauneuf pour les adapter à ces nouveaux bâtiments.

En 1978, les cargos fluviaux maritimes ont commencé à naviguer régulièrement sur le Bas-Rhône, et ont amené la C.N.R. à concevoir, pour l'écluse d'Avignon, un nouveau pare-chocs tenant compte à la fois des convois poussés et des cargos.

Pour les autres écluses, le problème des pare-chocs demande à être traité cas par cas selon les dispositions en place.

\section{Conception générale des nouveaux pare-chocs}

\section{Capacité énergétique}

Un convoi poussé de $11,40 \mathrm{~m}$ de large entrant dans une écluse de $12 \mathrm{~m}$ produit un effet de piston important et, pour se mettre en place, il doit conserver une certaine puissance à ses moteurs jusqu'à proximité du fond $d u$ sas, et battre alors machine arrière.

Pour préciser la valeur de l'énergie cinétique qu'il convenait de prendre en compte, la C.N.R. a procédé à des essais d'entrée de convois poussés, sur un modèle réduit au $1 / 50^{\mathrm{e}}$ de l'écluse de Bollene, construit dans son laboratoire d'hydraulique de Gerland.

De ces essais, on a pu tirer les conclusions suivantes : - la vitesse d'impact d'un convoi de $4 \mathrm{~m}$ d'enfoncement dont les moteurs sont maintenus à puissance constante semble plafonner à un peu plus de $1 \mathrm{~m} / \mathrm{s}$, quelle que soit cette puissance, du fait des mouvements du plan d'eau;
- l'effet de piston n'est important que sur les convois à pleine charge et semble localisé dans la section réđuite d'écoulement entre le fond du convoi et le seuil de la porte amont : la distance de freinage après l'arrêt des moteurs est de l'ordre de $15 \mathrm{~m}$ pour un convoi de $4 \mathrm{~m}$ d'enfoncement et de $100 \mathrm{~m}$ dès que son enfoncement n'est que de $3 \mathrm{~m}$;

- dans les essais de simulation d'entrée dite "normale", c'est-à-dire à vitesse progressivement décroissante, avec déclenchement de la marche arrière et arrêt du nez de la barge de tête à proximité du fond du sas, il semble que ce déclenchement doive intervenir à une distance comprise entre 60 et $30 \mathrm{~m}$ du point d'arrêt et que la vitesse normale lors de l'inversion de la marche des moteurs soit comprise entre 0,50 et $1 \mathrm{~m} / \mathrm{s}$.

Le relevé des vitesses des convois poussés après la première période d'adaptation des pilotes, a confirmé ce dernier résultat.

En conclusion, pour être en mesure de se garantir contre toute erreur de manœuvre, qu'elle soit humaine ou mécanique, la Compagnie a pris en compte une vitesse d'impact de $1 \mathrm{~m} / \mathrm{s}$, ce qui correspond à une énergie cinétique maximale de 3750 kilojoules pour une masse totale déplacée de 7500 tonnes correspondant aux convois circulant sur le Rhône.

Cette valeur de 3750 kilojoules correspond également à l'énergie d'un convoi lège arrivant en fond de sas à la vitesse de $2 \mathrm{~m} / \mathrm{s}$ environ.

\section{Distances d'arrêt}

Elles ne sont pas uniformes pour toutes les écluses, mais varient au contraire en fonction des dimensions des sas, de la configuration des installations et des systèmes adoptés.

\section{Ossatures}

Elles se présentent sous deux formes différentes : - soit deux poutres pare-chocs horizontales reliées par 2 boucliers verticaux;

- soit un tablier constitué d'une ossature plane caissonnée.

\section{Amortisseurs}

Les dispositifs sont constitués par des vérins hydrauliques ou des amortisseurs à compression hydrostatique d'élastomère.

\section{Description des nouveaux pare-chocs}

\section{Ecluse de Bollène (Fig. 1 et 2)}

La porte levante aval et le masque qui la prolonge, comme la porte baissante amont et le mur de chute furent réalisés semi-cylindriques.

En vue en plan, un convoi poussé est un rectangle de $190 \times 11,40 \mathrm{~m}$, à peine arrondi aux quatre angles. En conséquence, C.N.R. a adopté une forme plane pour le nouveau pare-chocs.

Le dispositif comporte deux poutres horizontales de $11,20 \mathrm{~m}$ de longueur disposées en travers du sas, aux cotes $(58,50)$ et $(62,50)$ NGF. 


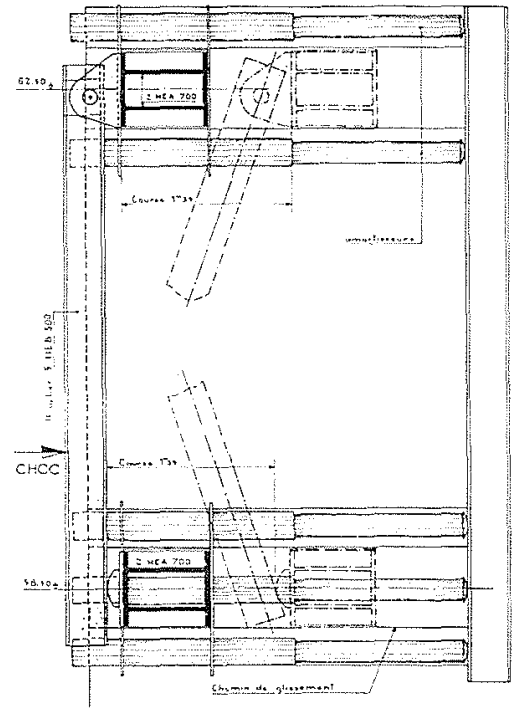

Figure 1 - Pare-chocs de l'écluse de Bollène.

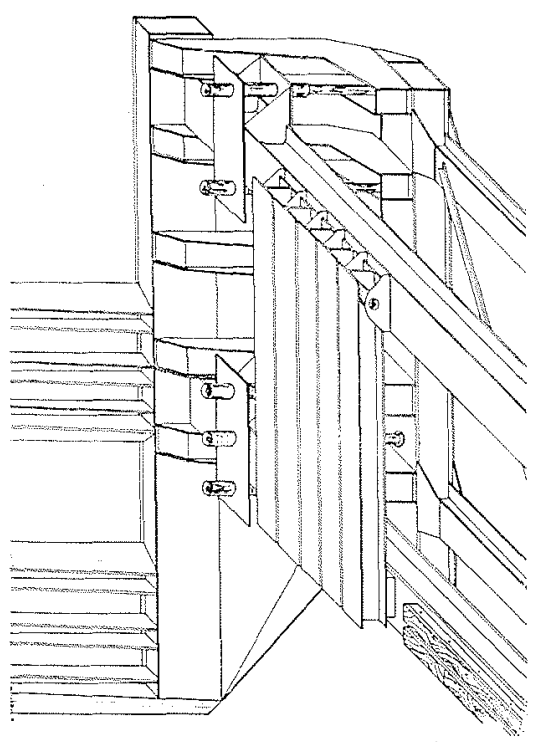

Figure 2 - Pare-chocs de Bollène

- la poutre inférieure, constituée de 2 HEA 700 soudés, porte 2 fois 3 amortisseurs de type BC-1S-60D système Jarret, à compression hydrostatique d'élastomère ; - la poutre supérieure, identique à la précédente, porte 2 fois 2 amortisseurs de même type.

Chaque amortisseur peut dissiper, de façon entièrement réversible, une énergie de 625 kilojoules pour une vitesse d'impact de $1 \mathrm{~m} / \mathrm{s}$ sur une course de $1,35 \mathrm{~m}$.

Entre les deux poutres horizontales d'amortissement, deux boucliers verticaux, articulés sur la poutre supérieure et appuyés sur la poutre inférieure permettent de reporter sur l'une ou l'autre de ces poutres les efforts de contact de la barge de tête quel que soit son tirant d'eau. Ils sont constitués de 5 HEB 300 .

La distance entre ces deux boucliers est de 7,20 m.

\section{Ecluse de Chateauneuf}

Le nouveau pare-chocs de l'écluse de Chateauneuf (portes semi-cylindriques) a été réalisé de façon analogue à celui de Bollène pour profiter de l'expérience acquise et d'une normalisation des matériels.

Le dispositif comporte également deux poutres horizontales de $11,90 \mathrm{~m}$ de longueur, disposées en travers du sas, aux cotes $(71,10)$ et $(81,50)$ NGF, et deux boucliers verticaux constitués de 5 HEB 360 .

- la poutre inférieure, constituée de 2 HEA 800 soudés, porte 2 fois 3 amortisseurs de type BC-1S-60D système Jarret, à compression hydrostatique d'élastomère ; - la poutre supérieure, constituée de 2 HEB 600 soudés, porte 2 fois 2 amortisseurs de même type.

La distance entre les deux boucliers est identique à celle de Bollène car il a paru important de normaliser, sur nos dispositifs pare-chocs, les points de contact avec les barges.

\section{Ecluse de Sablons (Fig. 3)}

Le pare-chocs aval installé au titre du marché a été calculé pour encaisser le choc d'un bateau possédant une énergie cinétique de 10 joules. Le dispositif amortisseur peut fonctionner pour les bateaux heurtant le pare-chocs jusqu'à une vitesse de $1 \mathrm{~m} / \mathrm{s}$. La course maximum des vérins amortisseurs est de $1,23 \mathrm{~m}$. Le tablier a la possibilité de pivoter selon le positionnement du point de choc.

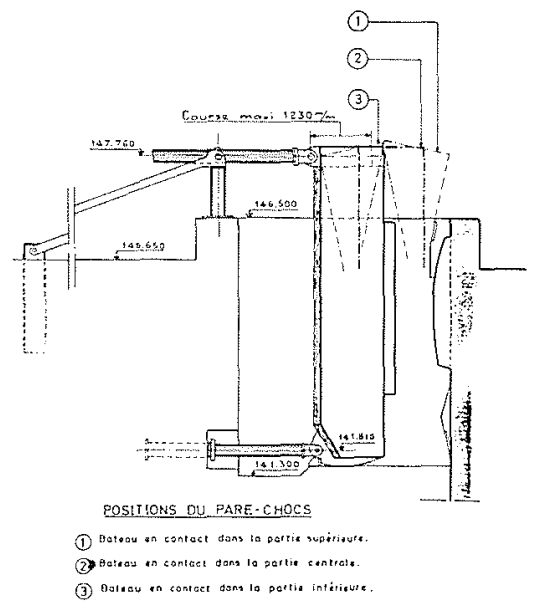

Figure 3 - Pare-chocs de l'écluse de Sablons

Le dispositif comporte essentiellement un tablier constitué d'une ossature plane caissonnée de $4 \mathrm{~m}$ de hauteur régnant entre les cotes $(141,65)$ et $(145,65)$ et des amortisseurs prévus pour absorber l'énergie d'un bateau et constitués par des vérins à élastomère.

\section{Ecluse de Vaugris (Fig. 4)}

Le pare-chocs aval a été calculé pour absorber une énergie cinétique de 3750 kilojoules correspondant à un convoi poussé de $7500 \mathrm{t}$ de masse totale, animé d'une vitesse de $1 \mathrm{~m} / \mathrm{s}$. Le déplacement maximal admis pour ce pare-chocs est de $2,36 \mathrm{~m}$.

Le dispositif comporte essentiellement :

- Un tablier constitué de deux ensembles mécanosoudés, d'une hauteur de $6,30 \mathrm{~m}$ régnant du niveau 148,50 au niveau 154,80 .

Par souci de simplification (suppression d'une paire 


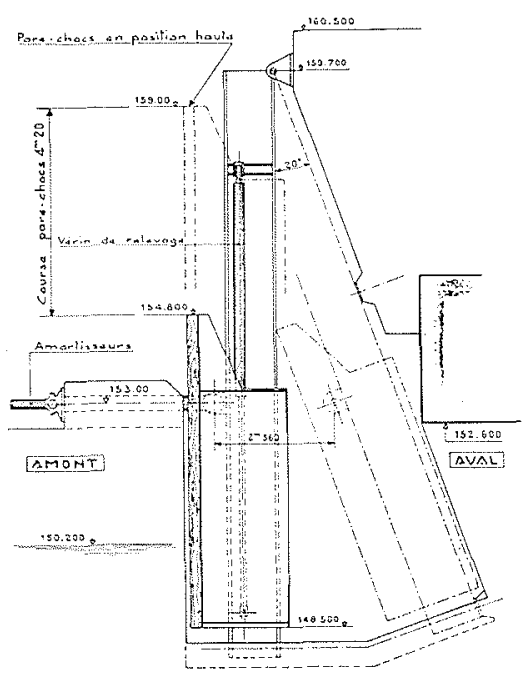

Figure 4 - Pare-chocs de l'écluse de Vaugris

d'amortisseurs), le mouvement de translation du tablier a été remplacé par un mouvement pendulaire.

Afin de pouvoir dégager le tirant d'air de $7,10 \mathrm{~m}$ audessus des PHEN, le tablier est également relevable. - Un ensemble de pièces fixes comprenant notamment des éléments de guidage du tablier articulés au niveau supérieur par des chapes fixées aux bajoyers de l'écluse - Deux amortisseurs à élastomère placés horizontalement, reliés par chapes articulées du type cardan, d'une part aux profilés de guidage du tablier, d'autre part aux bajoyers ;

- Un mécanisme de manœuvre comportant deux vérins hydrauliques qui assurent le relevage et la remise en position normale du tablier.

\section{Ecluse d'Avignon (Fig. 5 et 6 )}

Destiné à remplacer le pare-chocs actuel, dimensionné pour 100 kilojoules, le nouveau dispositif qui sera installé prochainement a été calculé pour dissiper une énergie maximum de 3750 kilojoules, correspondant à un convoi poussé de $7500 \mathrm{t}$ de masse totale, animé d'une vitesse de $1 \mathrm{~m} / \mathrm{s}$. Le déplacement maximal admis pour ce pare-chocs est de $1,35 \mathrm{~m}$.

Dans cette écluse, un problème particulier s'est posé du fait de la position du mirador (position basse en milieu de sas). I1 ne pouvait pas être envisagé un mouvement de rotation du tablier, en cas de choc de barge

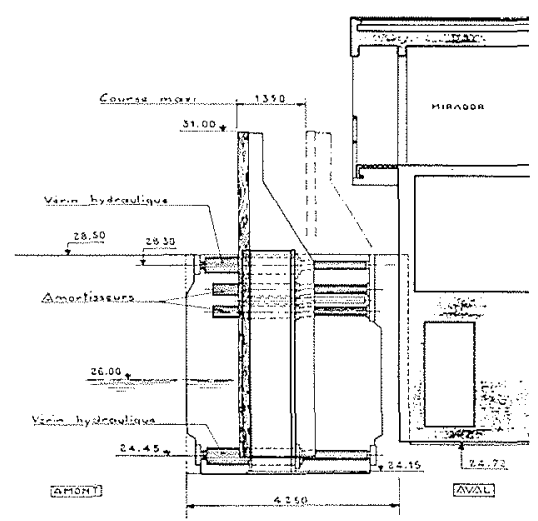

Figure 5 - Pare-chocs de l'écluse d'Avignon

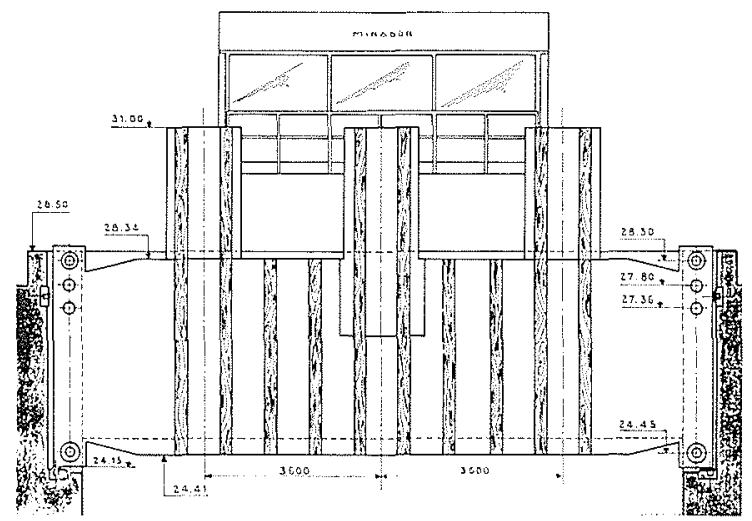

Figure 6 - Pare-chocs de l'écluse d'Avignon

lège, sans réduire de façon importante la longueur utile du sas.

Le nouveau pare-chocs a donc été étudié avec un asservissement hydraulique ne permettant que des mouvements de translation au tablier.

Le nouveau dispositif dont l'installation est prévue pour 1981 sera composé essentiellement de :

- un tablier sur lequel seront fixés trois boucliers verticaux (deux boucliers latéraux et un bouclier central). Le rôle des tabliers latéraux est d'arrêter les barges lèges dont la proue peut se situer au niveau $(30,50)$. Le troisième bouclier vertical fixé au centre du tablier a pour rôle d'arrêter certains bateaux lèges dont la proue de forme effilée peut se situer à un niveau supérieur à $(30,50)$;

- quatre amortisseurs de type BC.1S.100M système Jarret, à compression hydrostatique d'élastomère, montés sur le tablier. Chaque amortisseur pourra dissiper, de façon entièrement réversible, une énergie de 935 kilojoules pour une vitesse d'impact de $1 \mathrm{~m} / \mathrm{s}$ sur une course de $1,35 \mathrm{~m}$;

- quatre vérins double tige, montés également sur le tablier, qui devront assurer l'asservissement hydraulique de ce tablier.

\section{II - CHOCS SUR LES PILES DE PONT}

\section{Généralités}

Dans la définition des pare-chocs de piles de pont, nous avons supposé que cette protection était assurée par une glissière horizontale prenant appui sur des palpieux.

Nous avons effectué des études préliminaires et spécialement nous avons procédé à des essais sur modèle physique (échelle 1/33) afin d'étudier les comportements de la protection et du convoi lors d'un choc. Les points principaux à étudier étaient les suivants (Fig. 7) :

- angle optimal fait par la glissière avec l'axe longitudinal du canal ; 


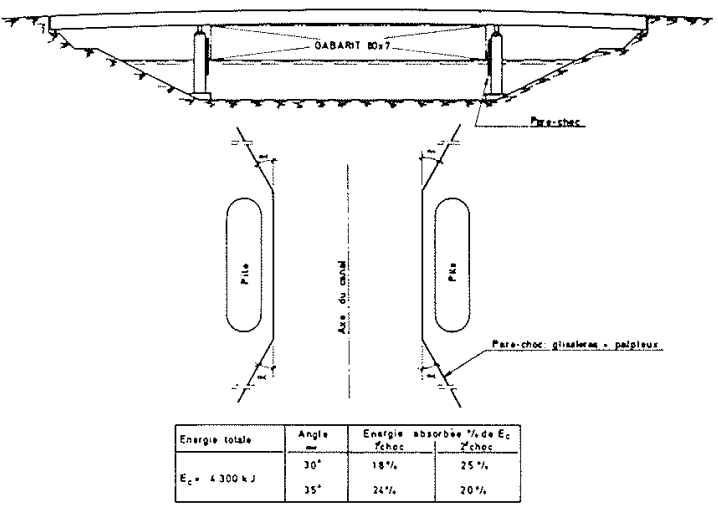

Figure 7

- énergie absorbée par le pare-choc;

- comportement du convoi après le choc.

En conclusion de ces essais, nous pouvons dire que : - l'angle optimal se situe aux environs de 30 à $35^{\circ}$. Pour des valeurs inférieures, la longueur de la glissière est trop grande, pour des valeurs supérieures le convoi est rejeté sur la pile opposée ;

- l'énergie absorbée par le pare-choc est égale à $50 \%$ environ de l'énergie totale ;

- pour une protection déformable le choc est assez long, le point de contact constitue un pivot pour le convoi qui effectue une rotation plus importante, une partie de l'énergie se dissipe donc en énergie de rotation.

Compte tenu de ces premiers résultats, dans l'étude plus détaillée des protections, nous avons classé les ponts en deux catégories :

- les ponts lourds c'est-à-dire les ponts dont les efforts verticaux sur les piles sont importants et de ce fait nécessitent des fondations importantes permettant de donner à celles-ci une structure capable d'encaisser les chocs de bateaux ;

- les ponts légers dont les piles ne peuvent apporter par la définition de leur structure une masse suffisante pour assurer la stabilité du pont sous l'effet d'un choc.

Dans la détermination des pare-chocs, les efforts pris en compte sont ceux de la circulaire $n^{\circ} 71-155 \mathrm{du}$ 29 Décembre 1971 à savoir :

- choc frontal : $8000 \mathrm{kN}$;

- choc latéral : $1600 \mathrm{kN}$.

\section{Ponts lourds (Fig. 8)}

Dans cette catégorie d'ouvrages, nous avons en exemple le pont assurant à la RN 519 le franchissement du canal de dérivation de la chute de Péage-de-

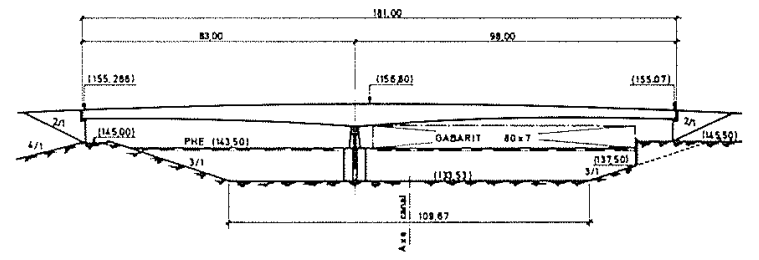

Figure 8 - Chute de péage de Roussillon RN 519
Roussillon (Rhône). Ce pont situé au droit de la zone portuaire de Sablons est un ouvrage dont la distance entre nus des culées est de $179,00 \mathrm{~m}$. Il comporte deux travées continues : $83,00 \mathrm{~m}$ et $98,00 \mathrm{~m}$; la travée de gauche située côté zone portuaire laisse libre un gabarit de navigation de $80 \mathrm{~m}$ de largeur et de $7,00 \mathrm{~m}$ de hauteur au-dessus du niveau maximal du plan d'eau.

Le pont est situé dans une plaine dont le substratum, constitué de marne pliocène, se trouve à un niveau très bas (vingt deux mètres environ sous le terrain naturel). La marne est recouverte d'une couche d'alluvions graveleuses dont l'épaisseur est de $12 \mathrm{~m}$ environ. Ces alluvions sont elles-mêmes recouvertes d'une couche de limon argileux et de terre végétale.

\section{Description de la pile (Fig. 9).}

L'ouvrage comporte une pile qui est constituée : - en partie haute, d'un fût en béton armé sur lequel viennent s'appuyer les caissons formant le tablier; - en partie basse d'un massif en béton ceinturé par un rideau de palplanches.

La réaction, sous poids propre du tablier, sur la pile étant de $4000 \mathrm{t}$, le massif en béton a été défini de façon à encaisser les chocs éventuels des bateaux, les contraintes au sol restant dans des limites acceptables pour des alluvions graveleuses.

Le massif comprend en section horizontale :

- une partie centrale rectangulaire de $21,8 \mathrm{~m}$ de longueur pour $6,10 \mathrm{~m}$ de largeur;

- des avant et arrière becs triangulaires de $6,10 \mathrm{~m}$ de largeur et $4,40 \mathrm{~m}$ de hauteur. Les côtés du triangle forment un angle de $35^{\circ}$ environ avec l'axe du canal; - l'assise du massif se trouve au niveau $(128,00)$ N.G.F. soit $5,50 \mathrm{~m}$ sous le plafond du canal. Ce niveau a été obtenu d'après le volume à donner au massif pour assurer la stabilité de la pile en cas de choc;

- le partie basse du massif est ceinturée par un rideau en palplanches descendu $10,00 \mathrm{~m}$ sous le plafond du canal permettant ainsi en cas de choc de mobiliser dans le terrain, une butée suffisante ;
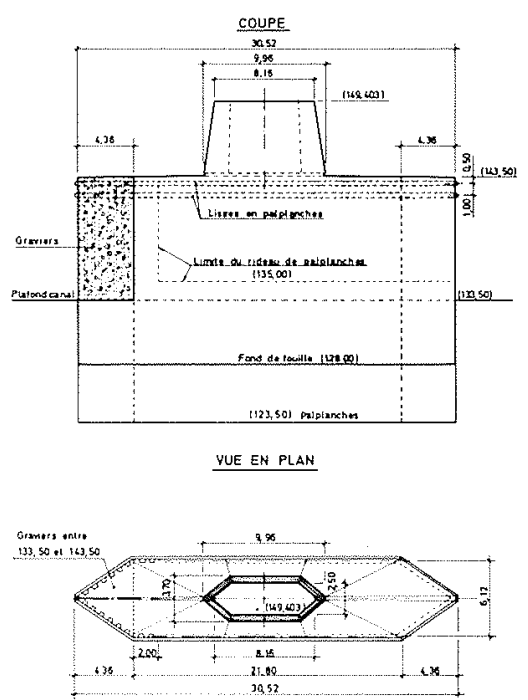

Figure 9 - Chute de péage de Roussilon RN 519 
- côté amont l'avant-bec est constitué d'un rideau de palplanches. L'espace triangulaire est empli de gravier compacté, l'ensemble formant ainsi une protection déformable, nous avons choisi les palplanches pour constituer l'enceinte triangulaire car ce matériau permet, en cas de choc, le remplacement facile des éléments inutilisables;

- la partie supérieure du massif est ceinturée par deux lisses en palplanches pour protéger le béton sous les chocs transversaux.

\section{Contraintes au sol}

Sous la semelle du massif de fondation, les contraintes maximales au sol sont les suivantes:

- poids propre

$0,48 \mathrm{MPa}$

- poids propre + surcharges

$0,57 \mathrm{MPa}$

- poids propre + choc frontal $(8000 \mathrm{kN})$

$0,76 \mathrm{MPa}$

Sous l'effet du choc frontal, la contrainte maximale atteint une valeur acceptable pour des alluvions graveleuses. Les contraintes au sol obtenues sous le choc transversal $(1600 \mathrm{kN})$ sont sensiblement identiques.

\section{Ponts légers (Fig. 10)}

\section{Généralités}

Dans la catégorie des ponts légers, nous présentons les ponts de la chute de Belley (Haut-Rhône) qui assurent à des voies communales et départementales le franchissement du canal de dérivation alimentant l'usine hydro-électrique de Belley. Le débit dérivé étant plus faible que celui de la chute de Péage-de-Roussillon, le canal présente des caractéristiques géométriques plus modestes.

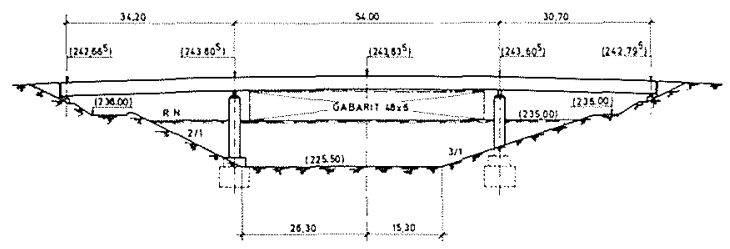

Figure 10 - Chute de Belley pont de la VC 10

Les ponts prévus sur ce canal sont des ouvrages de $120 \mathrm{~m}$ de longueur totale comportant trois traversées.

Sur cette partie du Rhône aménagée, il n'est pas prévu dans un premier temps de navigation commerciale; toutefois celle-ci pouvant se développer dans le futur, les caractéristiques des ouvrages ont été déterminées en tenant compte d'un éventuel trafic commercial.

Les piles centrales ayant une structure relativement légère, il était donc nécessaire de prévoir la réalisation ultérieure d'un pare-choc, protection indépendante du pont. Ce critère a donc, en fonction du gabarit de navigation $(40 \times 6 \mathrm{~m})$, permis de définir la portée de la travée centrale.

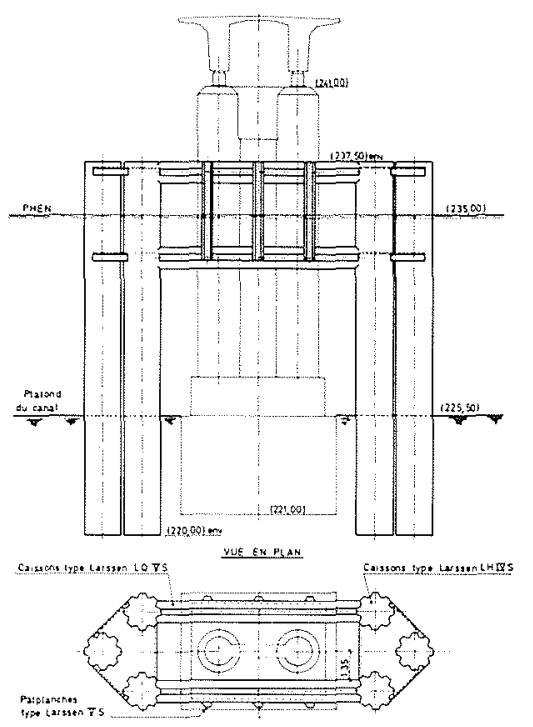

Figure 11 - Chute de Belley

\section{Définition de la protection (Fig. 11)}

Le dispositif anti-choc prévu en projet comprendrait : - à l'amont et à l'aval des piles, trois caissons en palplanches. Ces caissons seraient ceinturés sur leur périphérie par des liernes assurant la liaison entre les trois caissons ;

- les enceintes amont et aval seraient liaisonnées entre elles de part et d'autre des piles, par des traverses horizontales formant des poutres capables de résister au efforts transversaux et assurant la transmission des efforts frontaux à l'ensemble des caissons verticaux.

Les caractéristiques des caissons ont été déterminées de façon que l'ensemble amont-aval formant un portique soit capable d'encaisser l'effort longitudinal de $8000 \mathrm{kN}$.

La traverse horizontale du portique a été dimensionnée pour résister à l'effort transversal de $1600 \mathrm{kN}$.

L'encastrement des caissons verticaux dans le sol de fondation a été déterminé de façon que la butée mobilisée sur la hauteur de la fiche soit supérieure à l'effort provoqué par le choc.

Ce dispositif ainsi formé serait indépendant des piles ne leur transmettant aucun effort en cas de choc.

\section{Conclusion}

Pour les deux types de protection décrits, nous avons effectué les calculs de stabilité et de dimensionnement des ouvrages, sans tenir compte de l'énergie cinétique du convoi. Nous avons supposé que celle-ci était absorbée par la déformation de la coque du bateau, les efforts pris en compte étant ceux de la circulaire ministérielle.

Seuls des essais sur modèle réduit permettraient : - de contrôler le comportement d'un convoi en cas de choc;

- de mesurer l'énergie absorbée par le dispositif de protection; 
- de préciser les forces à prendre en compte dans la détermination des caractéristiques des dispositifs de protection.

\section{III - EVALUATION DES EFFORTS TRANSMIS A UNE PILE DE PONT AU COURS D'UNE COLLISION AVEC UNE BARGE}

\section{Introduction}

A l'heure actuelle la connaissance du comportement de l'obstacle et du navire lors d'un choc est encore insuffisante. Une évaluation correcte des efforts développés et de leur évolution au cours de la collision représente une condition préalable indispensable à un dimensionnement sûr des piles de pont et de tout autre ouvrage de protection.

Dans ce but,. le Service Technique Central des Ports Maritimes et des Voies Navigables du Ministère des Transports a donc passé un marché d'études avec l'Institut de Recherches de la Construction Navale. Ce marché a pour objet la réalisation d'une étude de l'effort transmis à une pile de pont par un navire au cours d'une collision.

Les différentes étapes de cette étude sont développées au cours des chapitres suivants.

Etude comparative de différents modèles mathématiques de la collision d'une barge contre une pile de pont.

\section{Présentation du problème}

Un problème de collision entre une barge et une pile de pont implique nécessairement la prise en compte de quatre éléments qui interagissent les uns avec les autres: barges, pile de pont, sol de fondation, eau environnante.

Différents modèles sont utilisables pour ces quatre sous-ensembles du système mécanique que constitue un bloc en cours d'évolution.

\section{Modèles de calcul}

\section{Barge}

\section{a) Modèle géométrique}

Deux modélisations par éléments finis ont été mises au point pour une barge de dimensions $76,5 \times 11,4 \times 3,9 \mathrm{~m}$ avec un chargement réparti de $2500 \mathrm{t}$.

Le modèle B3 est décrit entièrement à l'aide de 246 nœuds, soit 738 degrés de liberté, et comprend 138 éléments finis à deux dimensions de type plaque et 111 éléments finis à une dimension de type barre et poutre.

Un modèle mathématique de cette ampleur correspond en réalité à la taille maximale des problèmes qu'il

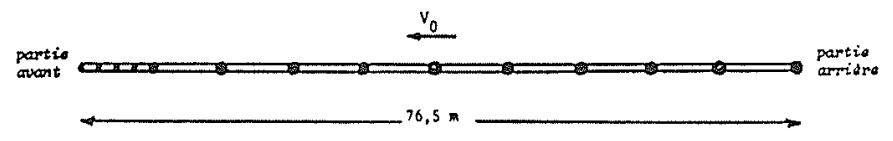

Modèle B 1 : représentation unidimensionnelle de la barge à l'aide d'éléments barre, conservant les sections réelles et ne travaillant qu'en traction-compression.

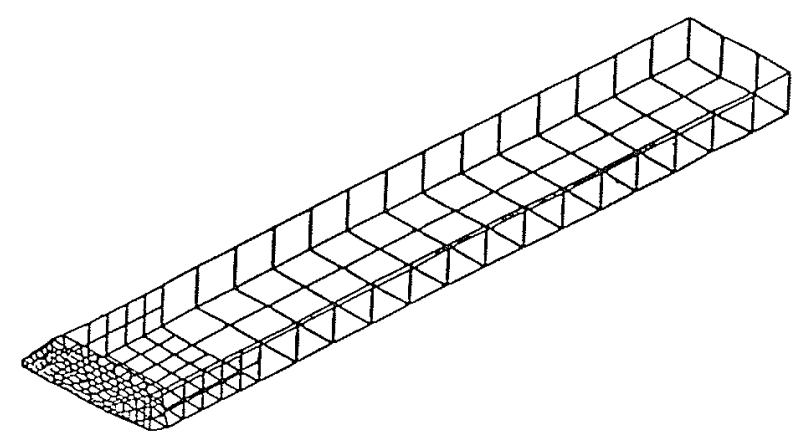

Modèle B 3 : Modélisation tridimensionnelle de la barge à l'aide d'éléments barre, poutre et membrane.

est actuellement possible de traiter en analyse non linéaire à l'aide du programme ADINA (programme du calcul de structures dans le domaine non linéaire, développé au Masachussets Institute of Technology par le Professeur KJ. Bathe, et utilisé dans le cadre de cette étude).

b) Modèles mécaniques

- Analyse linéaire : le matériau (acier) est supposé rester dans le domaine élastique linéaire durant toute la collision ;

On obtient ainsi les deux modèles linéaires B $1 \mathrm{~L}$ et B $3 \mathrm{~L}$;

- Analyse non linéaire : le matériau est supposé avoir un comportement élasto-plastique avec écrouissage isotrope.

On obtient ainsi les modèles B $1 \mathrm{NL}$ et B 3 NL.

\section{Pile de pont}

On a donc fait l'hypothèse d'une pile de pont absolument rigide, représentée uniquement par une masse ponctuelle $(M p=2000 t f)$ qui permet l'apparition des forces d'inertie à l'exclusion des forces de déformation élastique ou d'amortissement.

\section{Sol de fondation}

Une modélisation par un système discret équivalent (ressorts + amortisseurs) utilisée couramment en génie sismique, a paru suffisante. Le sol sera supposé se com-

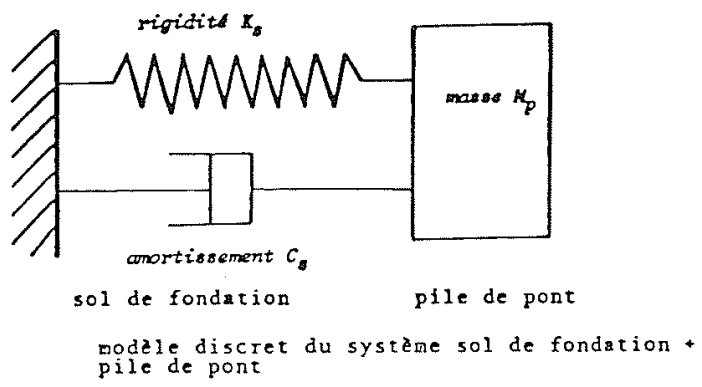


porter comme une série de ressorts de raideur $K s$ jusqu'à un seuil critique de déplacement $u_{c}$, au-delà duquel il ne supporte plus qu'une force constante $F_{c}$. La dissipation d'énergie due à l'amortissement visqueux interne au sol sera provoquée par la présence d'amortissement concentré $C_{s}$.

Dans le cas d'un sol moyennement compact, on a retenu les valeurs suivantes:

$$
\begin{aligned}
& K_{s}=10^{5} \mathrm{tf} / \mathrm{m} \\
& C_{s}=10^{3} \mathrm{tf} . \mathrm{s} / \mathrm{m}
\end{aligned}
$$

\section{Eau environnante}

La collision d'une barge contre une pile de pont est un phénomène essentiellement longitudinal, pour des angles d'incidence faibles. On est donc en droit de négliger, en première approximation, les effets des masses d'eau ajoutées, représentant l'influence de l'eau environnante et provenant des vibrations longitudinales de la barge.

En outre, la masse d'eau ajoutée varie dans le même sens que la durée de collision. Dans le cas d'un choc sur un obstacle rigide, cette dernière est très courte et donc la masse d'eau ajoutée est très faible.

\section{Résultats numériques et interprétation}

Dans le cas d'un choc frontal et pour une vitesse initiale de $10 \mathrm{~km} / \mathrm{h}$, l'intégration numérique pas à pas des équations de mouvement nous fournit en chaque instant l'accélération, la vitesse et le déplacement de chaque nœud du modèle $(\ddot{u}(t), \dot{u}(t)$ et $u(t))$ à l'instant $t$. On détermine leurs valeurs à l'instant $\mathrm{t}+\Delta t$ en supposant une variation linéaire de l'accélération entre les deux instants consécutifs.

A partir de ces résultats, les valeurs des différentes composantes de la force globale $F(t)$ qui s'exerce sur la pile de pont, ainsi que les énergies mises en jeu durant la collision sont calculées.

Les valeurs maximales de la force $F(t)$ pour les quatre modèles sont reportées dans le tableau suivant:

\begin{tabular}{|c|c|c|c|c|}
\hline Modèle & B 1 L & B 1 NL & B 3 L & B 3 NL \\
\hline $\begin{array}{c}F(t) \max \\
t f\end{array}$ & 17600 & 6800 & 6700 & 3100 \\
\hline
\end{tabular}

On ne peut que constater l'ampleur des écarts à la fois entre modèles linéaires et non linéaires, ainsi qu'entre modèles à une et trois dimensions. La solution a priori la plus vaisemblable correspond évidemment à la valeur la plus faible du modèle B 3 NL.

Les énergies calculées durant la collision ont été exprimées en pourcentage de l'énergie cinétique initiale $E_{0}$ de la barge $\left(E_{0}=1 / 2 M_{b} V_{0}^{2}=1100 \mathrm{t}\right.$ f.m).

Les valeurs extrêmes prises sont reproduites dans le tableau suivant :

\begin{tabular}{|c|c|c|c|c|}
\hline Modèle & B 1 L & B 1 NL & B 3 L & B 3 NL \\
\hline$\frac{E(t)}{E_{0}} \max$ & $94 \%$ & $62 \%$ & $56 \%$ & $35 \%$ \\
\hline
\end{tabular}

\section{Conclusions relatives aux différents modèles}

L'analyse des différents modèles possibles montre que :

- les modèles linéaires ne peuvent en aucun cas prétendre représenter la réalité du choc; les effets des non linéarités géométriques et du matériau deviennent prépondérants dès les premiers instants du choc.

- le modèle non linéaire à une dimension (B $1 \mathrm{NL}$ ) donne également des résultats supérieurs au modèle tridimensionnel (B $3 \mathrm{NL}$ ). Ceci provient vraisemblablement du fait que la conservation des sections réelles pour les éléments "barre", rend le choc beaucoup plus dur qu'en réalité.

Analyse non linéaire de trois types de choc

Pour étudier l'influence de la vitesse initiale et de la position d'impact liée à l'angle d'incidence, deux analyses non linéaires avec modèle tridimensionnel ont été effectuées pour les chocs correspondant au schéma suivant :

- Modèle B 3 NL 1 :

Point impact excentré, angle d'incidence de $15^{\circ}$, vitesse initiale de $10 \mathrm{~km} / \mathrm{h}$.

- Modèle B 3 NL 2 :

Point d'impact excentré, angle d'incidence de $15^{\circ}$, vitesse initiale de $5 \mathrm{~km} / \mathrm{h}$.

La méthode utilisée est en tout point identique à celle décrite dans la partie précédente.

Le tableau suivant donne les valeurs maximale et moyennes obtenues pour les trois chocs non linéaires, dans l'axe longitudinal de la pile.

\begin{tabular}{|c|l|c|c|c|c|c|}
\hline & & $\begin{array}{c}\text { Déplacements } \\
\mathrm{cm}\end{array}$ & $\begin{array}{c}\text { Vitesse } \\
\mathrm{cm} / \mathrm{s}\end{array}$ & $\begin{array}{c}\text { Accélération } \\
\mathrm{cm} / \mathrm{s} 2\end{array}$ & $\begin{array}{c}\text { Force } \\
\text { Tf }\end{array}$ & $\begin{array}{c}\text { Energie } \\
\text { Tf.m }\end{array}$ \\
\hline B 3 NL 0 & Max & 20,10 & 93,77 & 1330,06 & 3152 & 383 \\
& Moy ABS & 9,39 & 67,64 & 507,93 & 1911 & 224 \\
\hline \multirow{2}{*}{ B 3 NL 1 } & Max & 7,28 & 33,45 & 387,56 & 1560 & 99 \\
& Moy ABS & 3,04 & 24,56 & 123,71 & 1295 & 41 \\
\hline \multirow{2}{*}{ B 3 NL 2 } & Max & 3,40 & 16,22 & 352,65 & 1230 & 36 \\
& Moy ABS & 1,73 & 11,43 & 78,16 & 1040 & 17 \\
\hline
\end{tabular}


Comparaison globale : Il semble donc que le comportement d'une pile de pont au cours d'une collision soit moins sensible aux variations de la vitesse initiale qu'à un changement de la position du point d'impact et de l'angle d'incidence. Cette remarque s'applique particulièrement aux valeurs des forces maximales et moyennes communiquées par la barge à la pile de pont. Le tableau suivant donne les forces, obtenues avec les modèles B 3 NL 0 et B 3 NL 2, comme un pourcentage des valeurs obtenues avec le modèle B 3 NL 1 .

\begin{tabular}{|c|c|c|}
\hline Modèle & B 3 NL 0 & B 3 NL 2 \\
\hline$F$ max $/ F \max 1$ & $202 \%$ & $79 \%$ \\
\hline$F$ moy/F moy 1 & $148 \%$ & $80 \%$ \\
\hline
\end{tabular}

\section{Modèle simplifié}

Les modèles tridimensionnels non linéaires ( $\mathrm{Cf}$. Chapitres précédents) sont d'une utilisation assez lourde. Le but de cette dernière phase de l'étude a donc été de trouver une méthode d'identification permettant de passer de ces modèles à des modèles unidimensionnels équivalents en force et en énergie.

Le choix des paramètres soumis à la procédure d'identification a été dicté par des raisons physiques. La partie avant de la barge, modélisée par 4 barres non linéaires, apparaît comme la zone la plus sensible et par conséquent, les quatre sections de ces éléments ont été retenues. Par contre, la zone des cales reste quant à elle dans le domaine élastique et possède un comportement global. Ainsi les deux autres variables considérées sont la section de la zone des cales et la masse totale du chargement.

En résumé, il est donc possible d'obtenir avec un degré de corrélation suffisant, les coefficients de réduction permettant de passer du modèle tridimensionnel à un modèle unidimensionnel équivalent en force et énergie.

Cependant ce programme d'identification n'est utilisable que pour un angle d'incidence variant de 0 à $15^{\circ}$, pour une vitesse initiale de 0 à $15 \mathrm{~km} / \mathrm{h}$ et pour le type de barge précis. Néanmoins, il doit être possible, avec un degré de confiance raisonnable, de l'utiliser pour des structures similaires.

\section{Conclusion}

L'étude précédemment décrite, permet donc pour un choc de barge, avec un angle d'incidence de 0 à $15^{\circ}$ et une vitesse initiale de 0 à $15 \mathrm{~km} / \mathrm{h}$, de suivre l'évolution au cours du choc: du déplacement, de la vitesse, de l'accélération de la pile de pont, de la force totale transmise par la barge et de l'énergie reçue par la pile de pont.

On remarque que le modèle donne pour un choc frontal à $10 \mathrm{~km} / \mathrm{h}$ un effort maximal sur la pile de $3150 \mathrm{tf}$, soit beaucoup plus que ce qui est prévu par la circulaire de 1971 (800 tf). Mais la durée d'application de cet effort est très brève et il s'agit d'un cas extrêmement défavorable. En effet, la trajectoire de la barge est confondue avec l'axe de la pile et celle-ci a une face avant plane. Cette hypothèse aboutit à la meilleure répartition du choc sur la barge, d'où la déformation minimale de la barge.

Il reste une interrogation sur le mode de prise en compte de tels efforts, de caractère impulsionnel, dans la conception des ouvrages, les chocs étant, à l'heure actuelle, pris en compte sous forme de forces statiques.

Cette étude a donc permis la réalisation d'un outil indispensable qui est la modélisation de la barge. Le Service Technique Central des Ports Maritimes et des Voies Navigables envisage maintenant de prolonger cette étude en essayant de comparer de façon précise les efforts impulsionnels et les forces statiques, au niveau de leurs effets sur les piles de pont. 